\title{
Progress in research on the application of nursing models for breast cancer patients during the perioperative period $^{\dagger}$
}

Li-Ting Nie ${ }^{a}$, Qiao-Yuan Yan

a School of Nursing, Tongii Medical College, Huazhong University of Science and Technology, Wuhan, Hubei 430030, China

'Union Hospital, Tongji Medical College, Huazhong University of Science and Technology, Wuhan, Hubei 430022, China

Received: 7 August 2017; Accepted: 10 December 2017; Published: 20 June 2018

Abstract: Nursing models at home and abroad for breast cancer patients during the perioperative period were screened, including eight types of models: the nursing model guided by self-care theory, the plan-do-check-act cycle combined with the four-in-one model, the peer support nursing model, the nursing model guided by transcultural theory, the multidisciplinary cooperative nursing model, the knowledge-attitude-practice nursing model, the safe nursing management model, and the case nursing model. These models were analyzed and described with the aim of providing a reference for the clinical breast surgery nursing staff in China and for promoting the development of nursing in China for breast cancer during the perioperative period.

Keywords: Nursing models • breast cancer • perioperative period • literature review

(C) Shanxi Medical Periodical Press.

\section{Introduction}

Breast cancer has one of the highest incidences of cancer for women in the world, with approximately 1.67 million new cases of breast cancer and approximately 520,000 people dying of breast cancer each year; the incidence is the highest in developed countries. ${ }^{1-3}$ In China, approximately 250,000 women suffer from breast cancer each year, listed at the first for urban and rural female cancer patients; approximately 60,000 women die of breast cancer each year, listed at the sixth in lethality for women. These statistics indicate that although the incidence of breast cancer in women is high in China, the mortality is relatively low. At present, breast cancer is primarily treated

${ }^{\dagger}$ This project was supported by a scientific research project of the Hubei Province Health and Family Planning Commission, China (No. WJ2017M100). with surgery, and its 5-year observed survival rate is approximately $72.7 \%{ }^{4}$ The high incidence and low mortality of breast cancer are challenges to the nursing staff. Supportive nursing is the core of cancer treatment; it involves not only immediate treatment ${ }^{5}$ but also tries to solve a series of problems, including those in the physiological, psychological, social, and spiritual realms, for the patients, their family members, and caregivers. Many nursing models have been proposed at home and abroad in connection with nursing for breast cancer during the perioperative period, and they have all played an important role in clinical application; however, there are comparatively few studies in the literature summarizing the status quo of research on the various types of nursing models. Considering this lack, progress in research at home and abroad on the application of nursing models for breast cancer patients during the perioperative period is summarized in this paper, with the aim of providing a reference for 
the clinical breast surgery nursing staff in China in the selection of a nursing model and for promoting the development of the nursing profession in China for breast cancer during the perioperative period.

\section{Status on the application of nursing models for breast cancer during the perioperative period}

Because breast cancer treatment is mainly based on surgery, the nursing issues that need attention during the perioperative period include postoperative limb function recovery; prevention of postoperative complications; self-image reconstruction; improvement in cancerrelated fatigue; regulation of anxiety, depression, and other emotional disturbances; elimination of "uncertainty in illness"; and reduction of side effects from cultural shock on disease rehabilitation, among others. In connection with these nursing issues, there are many nursing models in the world related to breast cancer during the perioperative period, including nursing models provided by professional breast care nurses, general practitioners, peer support groups, and cancer support agencies and councils, along with the most recent nursing support model provided through virtual platforms, with the aim of providing better help to breast cancer patients during the perioperative period. For example, Orem's self-care model has a significant advantage in terms of increasing the self-care capacities of breast cancer patients; the peer support model is able to better reduce the patients' negative emotions; the plan-do-check-act (PDCA) cycle combined with the four-in-one model is able to continuously increase the patient self-care behavior and quality of life; the transcultural nursing model effectively lowers the cultural maladjustment of foreign breast cancer patients; the multidisciplinary cooperative nursing model maximally promotes fast rehabilitation in patients; the knowledge-attitude- practice (KAP) nursing model effectively increases compliance in breast cancer patients; the safe management model makes breast cancer nursing precise and refined; and the case management model rebuilds patients experiencing uncertainty in illness. Thus, each nursing model has a point of emphasis, and the study of nursing models for breast cancer patients during the perioperative period is ongoing. However, although the many nursing models have different names, their contents can overlap significantly. After careful reading, screening, and comparing, eight types of more scientific nursing models are listed and described herein.

\section{Nursing models for breast cancer patients during the perioperative period}

\subsection{Nursing model guided by the self-care theory}

In 1971, the U.S. nursing scholar Orem proposed the self-care theory, which was introduced to China in 1983 by Shiai Lv and became an important model for clinical nursing in China. ${ }^{6}$ Orem believed that the nursing system is a behavioral system for developing openness and that the bases for selecting the nursing system are a patient's self-care need and self-care ability. When self-care deficits are formed so that the patient is unable to either partially or completely unable to perform continuous and effective self-care, nursing staff are needed to provide care and assistance, and they are asked to provide wholly compensatory nursing, partly compensatory nursing, or supportive education to the patients - based on their specific situations - to meet patients' self-care needs. Research has shown that in a nursing model under Orem's self-care theory, the incidence of postoperative complications for breast cancer patients was significantly reduced, the patients' limb function rehabilitation rate increased, and the awareness rate of health education knowledge was significantly higher than those of the conventional nursing group. ${ }^{7} \mathrm{Xu}^{8}$ used postoperative breast cancer patients in the Henan Province region of China as a sample and divided that sample into control and conventional groups. A conventional surgical nursing model was used for the control group, while a nursing model from Orem's self-care theory was used for the study group; in other words, based on whether the patients were in a lucid interval after surgery and their capacities for self-activity, a patient who was not lucid after surgery was listed as being in a wholly compensatory system, while one who was lucid after surgery and had the capacity for selfactivity was listed as being in a partly compensatory system, with emphasis placed on penetrating the full course of the perioperative period with supportive education. $\mathrm{Lu}^{9}$ especially emphasized paying attention to unleashing the patients' maximum self-care potential when applying this model, which requires the nurses to use their own wisdom in combination with the patients' physiological and psychological conditions that have been brought to bear, posing higher requirements on clinical nurses. Zhang ${ }^{10}$ assigned patients to three major systems according to the patients' postoperative chronology and believed that the wholly compensatory 
system-partly compensatory system-supportive education system is in a state of dynamic change, not isolated from each other; and the "self-care is a need of self-esteem" viewpoint was applied to the supportive education system. In a traditional nursing model, the patient passively accepts the help of the nursing staff, but in the self-care theory, the patient actively participates in decision-making regarding their health, with the objective of promoting self-care. It can be seen from this discussion that Orem's self-care theory starts with self-care deficits in postoperative breast cancer patients based on changes in postoperative disease conditions. The nurses can use a self-care ability assessment table to perform assessments, lightening their workload while allowing for improved targeting of postoperative nursing issues. As a full-course system, the supportive education system seems to be especially important in the course of nursing breast cancer patients. Because the majority of the patients are women and surgery can greatly change the beauty of the woman's original body shape, coupled with the great importance that women place on their own image, educating patients on psychological and physiological health throughout the process may promote postoperative rehabilitation and meet the demands of high-quality nursing care in modern times. However, Orem's self-care theory is limited to existing nursing diagnosis and is relatively lacking in research on stimulating factors.

\subsection{PDCA cycle combined with a four-in-one model}

The PDCA cycle proposed by the U.S. quality management scholar Deming is a basic method used in quality management. This cycle performs quality management based on the sequence of plan, do, check, and act; the starting point for each cycle is raised to a new level, and the cycle continues without end. ${ }^{11}$ The four-in-one model is from the joint participation of the physicians, nurses, patients, and family members in formulating a functional exercise pathway table and in gradually implementing the proper sequence according to the table, while following scientific and systematic principles to enhance the relevance and purpose of rehabilitation training by fully mobilizing the patients' initiative and enthusiasm. In particular, listening to other patients' testimonials in the synchronous training for family members lets family members grasp the relevant rehabilitation training, which is favorable for supervising and increasing patient interest in functional exercises and their effects. ${ }^{11}$ Ding and Wang ${ }^{12}$ used breast cancer surgery patients as study subjects. A traditional method was used with the control group, i.e., the responsible nurse conducted rehabilitation knowledge education during the perioperative period, and the remainder of the time was devoted to random rehabilitation education, mostly in a lecture format to guide a single patient; the patient would practice on her own after being instructed. The PDCA cycle combined with the four-in-one model was implemented with the observation group by creating a PDCA functional exercise group, and the patients were educated on the drafted four-in-one format functional exercise clinical pathway table. Existing problems were reviewed and analyzed at each execution, and the problems were combined with reality to then revise the pathway table for reimplementation; the cycle was then repeated until the patients were rehabilitated. Research has shown that the PDCA cycle combined with the four-in-one model effectively solves the nurses' "oneperson platform" situation in traditional rehabilitation guidance, promotes exchange and cooperation between medical and nursing staff, increases enthusiasm in the patients and family members, and is able to continue to improve the quality of nursing, thereby effectively ensuring patient rehabilitation. Of course, this model also poses very high requirements on the nurses' specialized knowledge; requires the nurses to have very good capabilities in analyzing, judging, summarizing, and applying knowledge; and may require them to play a leading role in this group of physicians, nurses, patients, and family members.

\subsection{Peer support nursing model}

The peer support model is a type of nursing model that provides help to physically and psychologically healthy patients under treatment during the rehabilitation period. ${ }^{13}$ In other countries, two methods are used: face-to-face and virtual platforms. Research has shown that this model is able to effectively promote the psychological health of patients and to reduce adverse reactions during the perioperative period; sharing life experiences and professional lifestyles has been demonstrated to be able to reduce anxiety and a sense of loneliness in patients and to help improve their quality of life. ${ }^{5}$ Cao et al. ${ }^{14}$ divided 60 patients at different time periods at a hospital into control and study groups. Conventional nursing was implemented for the control group, while for the study group, a peer support model was used on the basis of conventional nursing to organize and guide volunteers and to provide help to patients during the preoperative and rehabilitation periods; the timing was once per week, and the contents were rehabilitation experience and emotional support. 
While the breast cancer patients' physical and psychological health parameters were effectively improved and postoperative complications were reduced through 2 months of intervention, there were also great savings in health-care human resources. Borkman ${ }^{15}$ proposed the "concept of being similar to this person." This concept deems that empathy from patients who had similarly suffered and were subsequently rehabilitated from cancer using their firsthand experience and knowledge to provide support and help to patients with the same disease can make other patients look at the disease from a unique perspective and obtain social support. Dunn et al. ${ }^{16}$ also performed the same research and demonstrated that the peer support model could significantly reduce the sense of loneliness in patients and can provide patients with sufficient confidence to overcome their disease. However, the peer support model can only be an auxiliary nursing model for breast cancer patients during the perioperative period because the nursing staff are still playing the leading role in promoting patient rehabilitation. ${ }^{20}$ Moreover, the peer support model requires a hospital or an organization to establish standardized breast cancer volunteer institutions and also needs the regular input of medical staff for their training; only after they have met training standards can they enter the hospitals to help patients suffering from the same disease. Therefore, it is more suitable for use in urban areas where the volunteers are of higher quality and the level of medical treatment is developed and abundant. Patients will be limited in their participation in face-to-face peer support after they have completed treatment, recovered, and been discharged from the hospital. Emerging virtual platform services supplement face-to-face support, reduce the burden on the health-care system, and prevent patients and volunteers from rushing back and forth. ${ }^{17}$

\subsection{Nursing model guided by the transcultural theory}

The transcultural nursing theory was first proposed by the U.S. nursing scholar Madeleine Leininger and was introduced to China in 1995. The "Sunrise Nursing Mode" (SNM) is the core of the transcultural nursing theory. SNM deems nursing as providing differentiated care to patients with different ethnic and cultural backgrounds in a targeted manner within a relative framework, rather than a fixed model. ${ }^{18}$ Wang et al. ${ }^{18}$ implemented a nursing model guided by transcultural theory on 10 foreign breast cancer patients during their perioperative periods at the hospital. ${ }^{19}$ After the patients were admitted to the hospital, they were assessed in accordance with SNM's three-level assessment method.
The first level assessed the patients' beliefs, educational backgrounds, languages, and personal economic status. The second level assessed the patients' health, their caregivers, and medical expenses. The third level assessed the patients' health needs, treatment needs, and nursing needs. Based on the assessment results, contents were formulated to compensate for the language communication barriers in nursing, psychological care, life care, and perioperative visit instructions, and specific nursing interventions were then performed. Research ${ }^{19}$ has shown that a nursing model guided by the transcultural theory avoids the occurrence of cultural coercion and culture shock due to cultural differences, improves the quality of nursing for foreign patients, carries forward the human-oriented spirit, and conforms to the world trend of global integration.

\subsection{Multidisciplinary cooperative nursing model}

In recent years, an important medical diagnostic model has appeared internationally: the multidisciplinary team (MDT), which promotes the joint development of multiple disciplines mainly by optimizing diagnosis and treatment methods, reasonably allocating medical resources, and improving diagnosis and treatment results. ${ }^{21}$ The MDT is often a multidisciplinary and interdisciplinary approach that includes not only surgical and medical teams but also breast care nurses, physical therapists, expert peer support, consultants, and psychologists. ${ }^{22}$ This model centers on the breast cancer patient and uses a multidisciplinary approach to take care of them and their families and has always been recognized by physicians, nurses, allied health teams, and the women themselves. ${ }^{23}$ Zhang ${ }^{21}$ divided his hospital's 120 breast cancer patients during their perioperative periods into observation and control groups. The hospital's conventional nursing model was used for the control group, while an MDT was established for the observation group. The first-level members included a breast surgeon, a specialist nurse, and a bed nurse, and the second-level members included physicians from nutrition, psychology, rehabilitation, plastic surgery, imaging, pathology, traditional Chinese medicine, and other departments, wherein the specialist nurse assumed the duties as the team's liaison officer. When the MDT was operating, there were two consultation meetings each week that first-level members must attend; second-level members may attend in accordance with the actual situation. The principles of coordination, mutual aid, and each giving full play to his/her strong points were followed, and on-the-spot assessment and analysis of the patients' disease conditions and pathological test 
results were performed, helping the nurse to follow a systematic nursing program. Throughout the nursing process, the patients received the most convenient and effective medical services, and repeat appointments for visits by patients in various departments were avoided, thereby shortening the patients' hospital stays. The results showed that the MDT nursing model is able to reduce the stress response of breast cancer patients during the perioperative period, reduce postoperative complications, and accelerate postoperative rehabilitation. Existing nursing issues for breast cancer patients during the perioperative period involve multiple disciplines; therefore, the use of a multidisciplinary cooperative nursing model can provide patients with the most professional medical resources. Moreover, the nursing staff who becomes the liaison officer not only must take part in the formulation of the nursing program but is also the main implementer of this program; the requirements for the nursing staff in terms of various capabilities are very high. For the physicians, two consultation meetings each week are also a challenge. At present, regular consultation meetings would be held for difficult illnesses in a grade A tertiary hospital, but regular consultation meetings for each breast cancer patient have not been implemented; moreover, nurses do not participate very much in consultation meetings, ${ }^{24}$ mainly due to the serious lack of domestic medical resources, the lack of manpower in terms of nurses, and fewer highly educated nurses. ${ }^{25}$

\subsection{KAP nursing model}

The KAP nursing model is an abbreviation of the factors knowledge $(K)$, attitude $(A)$, and practice $(P)$. The KAP theory was first proposed by the team of a professor of Harvard University, Mayo et al., in the United States and was later further developed by researchers and successfully applied in promoting changes in people's health behaviors. ${ }^{26}$ The KAP nursing model refers to enhancing the patients' mastery of knowledge about the disease by educating patients on health, gradually forming healthy beliefs, with the objective of enabling the patients to form positive, beneficial health behaviors in daily life. Lv $^{27}$ divided 58 breast cancer patients at her hospital into control and observation groups. Conventional nursing was implemented for the control group. The KAP nursing model was used on the basis of conventional nursing for the observation group. Educators for the KAP model were selected and trained to perform various forms of health education for breast cancer patients during the perioperative period. The contents mainly included explanation of the necessity of radical surgery and the postoperative rehabilitation process, making up for self-image disorders through breast reconstruction surgery, information on the inducing factors for breast cancer, and so on. Mapping breast cancer-related knowledge deeply into the minds of patients to gradually form beliefs exerts a subtle influence on the manifestation of favorable behavior for disease rehabilitation. Research ${ }^{27}$ has shown that as a method of continual health education, this model effectively increased the compliance of breast cancer patients during the perioperative period, thereby making them actively cooperate with treatment; the overall health assessment of the patients in the observation group was significantly higher than that of the control group. Compared with traditional health education, the KAP model emphasizes feedback from information recipients and whether the patients understand and are able to apply the knowledge transferred to them by the nurses and emphasizes the positive effects of health education on patient behavior; ${ }^{27}$ therefore, the model is worth promoting broadly in clinical use. However, the implementation effect of this model is related to the nurses' education, experience, and professional attitude.

\subsection{Safe nursing management model}

The safe management model refers to strict mastery of a variety of rules and regulations and operational procedures so that patients obtain physical and psychological safety during the perioperative period. Along with an increase in patient awareness of selfcare and protection of rights, running the concept of the safe nursing management model throughout the perioperative period for patients has already become a requirement of clinical nursing. Tastan et al. ${ }^{28}$ strictly followed the clinical pathway in nursing patients after breast cancer surgery and found that under this model, the patients' sense of anxiety was reduced, satisfaction was increased, and quality of life was better. $\mathrm{Wu}^{29}$ divided 120 breast cancer patients during the perioperative period at their hospital into a treatment group and a control group. Conventional nursing was used for the control group. A safe nursing management model was used for the treatment group. This model consisted of two parts, safe rehabilitation exercise and safe nursing quality management; the detailed description of safe rehabilitation exercises safely and effectively guided patients in postoperative rehabilitation training, and safe nursing quality management required the nursing staff to make work standardized and programmed. While the safe nursing model promotes the overall rehabilitation of patients, it also nurtures the nursing staff's own safety awareness and service awareness. ${ }^{28}$ 


\subsection{Case nursing model}

The case management model first appeared in social work management in the United States and was subsequently introduced to the medical and nursing fields in the United Kingdom and gained rapid development. In European and American regions, the breast cancer case management model is already quite mature. ${ }^{30}$ At present, China's nursing staff have started to pay attention to the application of case management in breast cancer patients, but there are still few relevant studies. Peng ${ }^{31}$ implemented a case management model for 40 breast cancer patients accepted for treatment at her hospital and compared them to 40 cases for whom a primary nursing model was implemented. A case management team was formed, and a case manager with $>8$ years of seniority, a bachelor's degree, and qualifying capabilities was selected. The contents of case management included recording patient information, formulating nursing plans, supervising and guiding patients by stage, and continuing nursing measures (telephone follow-up visits, establishing $Q Q$ group (a multiplayer communication service launched by Tencent), and announcing the case manager's telephone). During the breast cancer perioperative period, one-on-one full-course nursing was implemented and combined with primary holistic nursing to ensure there were no gaps throughout the entire nursing process. In particular, the case manager was in close contact with patients until 6 months after the patients had been discharged from the hospital and accepted telephone consultation from patients thereafter. Research ${ }^{31}$ has shown that the case nursing model not only unleashes the strengths of capable nursing staff while optimizing nursing resource allocation but also, to a great extent, eliminates the breast cancer patients' uncertainty in illness during the perioperative period, increasing patient satisfaction. However, there are also studies that have shown how reexaminations and follow-up visits would deepen the patients' sense of anxiety due to worry about breast cancer relapse. ${ }^{32}$ Moreover, relapses are mostly found by the women themselves, and they believe that participation in follow-up visits is unable to provide them with better ways to adjust to postcancer life; even though the hospital provides them with prescription drugs, there is no prescription that can make them healthier. ${ }^{33}$

\section{Outlook}

Breast cancer has the characteristics of high incidence and high survival rate, prompting researchers at home and abroad to study breast cancer nursing models during the perioperative period. More than 20 nursing models have been proposed in the past 5 years. Each nursing model has added new characteristics on the basis of conventional nursing to obtain differences in effect. Nursing models not listed in this paper, such as the comfort nursing model put forward by Wang ${ }^{34}$ and which implemented comfort nursing for breast cancer patients during the perioperative period, included the aforementioned and listed eight nursing models, which is also the reason why they are not independently listed in this paper. For the same reason, the primary nursing model, holistic nursing model, solution-focused model, cancer-related fatigue nursing intervention model, rapid rehabilitation model, full-course humanistic nursing model, clinical nursing pathway model, and systemic nursing model, among others, have been reflected in the contents of the aforementioned and listed nursing models. At present, the situation in China for using nursing models to target breast cancer during the perioperative period is relatively good, but there are comparatively few methods that combine multiple types of nursing models for use in breast cancer during the perioperative period. Liu et al. ${ }^{35}$ combined the individualized nursing model with universalization to achieve dualtrack continual health education, i.e., using the individualized nursing model and applying a continual nursing model, and obtained good clinical effect, which provided a reference for future research on better nursing models for breast cancer during the perioperative period. In Europe and Australia, there is a professional breast care model that is a type of autonomous and complementary supportive nursing service model, and breast health nurses are widely recognized, but this role is uncommon in China. In the United Kingdom, there is a supportive nursing model called "Advance," which consists of professionally trained breast care nurses; it is used to perform day-to-day follow-up visits with nurses at low-to-moderate risks, not for providing supportive nursing to patients, through a global positioning system (GPS) sharing function. ${ }^{36}$ The GPS effect model has replaced conventional follow-up visits and educational programs, providing a more cost-effective shared resource while also addressing patients' anxiety and worries after having cancer. These worries include menopausal issues, sexual issues, fear of relapse, and lymphedema, among others. This model is also combined with the companion care model, inviting the participation of female companions. ${ }^{19}$ In Victoria, there is an emerging nursing model to train professional breast care nurses at advanced levels of operation to meet the diverse needs of women diagnosed with breast cancer; the difference relative to an ordinary nurse is that they not only provide illness management but also pay attention to promoting the 
patient's health. ${ }^{37,38}$ Currently, nursing models operating through the Internet, e-mail, Skype, and smart phone apps to provide patients with support information are able to promote patient psychological and physiological health. ${ }^{17,39}$ An electronic solution consisting of a centralized cloud database to share public information and backup data with contents involving exercise, healthy eating, fatigue management, sleep issues, menopausal issues, cancer prevention, and screening settings, along with an emphasis on self-efficacy, is able to help women control their health. Users can use online videos for personal consultations with experienced cancer care nurses, and ongoing tests have shown gratifying results. ${ }^{40}$ It can be predicted that as the overall level of the nursing staff improves, nursing work for breast cancer during the perioperative period will also improve. A variety of nursing models may be used in corresponding situations, bringing good news to breast cancer patients while also enabling optimization of resource allocation at the hospital.

\section{Conflicts of interest}

All contributing authors declare no conflicts of interest.

\section{References}

1. DeSantis C, Ma J, Bryan L, Jemal A. Breast cancer statistics, 2013. CA Cancer J Clin. 2014;64:52-62.

2. DeSantis $C$, Siegel R, Bandi $P$, Jemal A. Breast cancer statistics, 2011. CA Cancer J Clin. 2011;61:409-418.

3. Mettlin C. Global breast cancer mortality statistics. CA Cancer J Clin. 1999;49:138-144.

4. Chen WQ, Zheng RS. Incidence, mortality and survival analysis of breast cancer in China. Chin J Clin Oncol. 2015;42:668-674.

5. Reed E, Scanlon K, Fenlon D. A survey of provision of breast care nursing for patients with metastatic breast cancer - implications for the role. Eur J Cancer Care (Engl). 2010;19:575-580.

6. Li XM. Introduction to Nursing. Beijing: People's Medical Publishing House; 2013:258-265. (in Chinese).

7. Xu M. Application of Orem self-care theory in postoperative breast cancer patients. China Med Engineer. 2016;24:111-112. (in Chinese).

8. Xu M. Study on nursing care of patients with breast cancer after operation using orem. China Contin Med Educ. 2016;8:248-249. (in Chinese).

9. Lu P. Application of orem self-care theory in the nursing of postoperative breast cancer patients. J Qiqihar Univ Med. 2014;35:2619. (in Chinese).

10. Zhang $X Q$. Current status on the application of orem self-care theory in the nursing of breast cancer patients. Nurs Pract Res. 2012;9:109-110. (in Chinese).

11. Wei BR. Application of PDCA Cycle-based process management in the graduation theses of nursing undergraduates. Chin J Nurs Educ. 2010;7:264-265. (in Chinese).

12. Ding J, Wang H. Application of PDCA cycle and four in one model in the limb function rehabilitation after breast cancer surgery. Chin J Modern Nurs. 2016;22:951-954. (in Chinese).
13. Qiu JJ, Hu Y, Huang JL, et al. Practice and evaluation of a volunteer peer support program for patients with breast cancer. Chin J Nurs. 2008;43:690-693. (in Chinese).

14. Cao XY, Deng SF, He LH. Effect of the patient mutual help-oriented nursing model on mental health and treatment-induced adverse reactions in breast cancer patients. J Nurs Sci. 2013;28:23-25. (in Chinese).

15. Borkman TJ. Experiential, Professional, and Lay Frames of Reference. Working with Self-help. Silver Spring, MD: NASW Press; 1990:3-30.

16. Dunn J, Chambers SK, Occhipinti S, Wilson KL. Evaluation of a peer support program for women with breast cancer = lessons for practitioners. J Community Appl Soc Psychol. 1999;9:13-22.

17. Meneses K, McNees P, Azuero A, Loerzel VW, Su $X G$, Hassey L. Preliminary evaluation of psychoeducational support interventions on quality of life in rural breast cancer survivors post-primary treatment. Cancer Nurs. 2009;32:385-397.

18. Wang L, Tan HQ, Yang N, et al. Application of cross-cultural nursing theory to foreign breast cancer patients in perioperative stage. J Nurs Sci. 2014;29:41-43. (in Chinese).

19. National Breast Cancer Centre. Specialist Breast Nurse Competency Standards and Associated Educational Requirements. Camperdown, NSW: National Breast Cancer Centre; 2005.

20. Stevinson C, Lydon A, Amir Z. Characteristics of professionally-led and peer-led cancer support groups in the United Kingdom. $J$ Cancer Surviv. 2010;4:331-338.

21. Zhang M. Application of multi-disciplinary teamwork nursing mode in perioperative care to breast cancer patients. Modern Clin Nurs. 2016;15:24-27. (in Chinese). 
22. Supportive Cancer Care Victoria. Supporting the Integrated Cancer Services to Implement the Supportive Care Policy for Victoria Supportive Cancer Care Victoria. http://www.supportivecancercarevictoria.org/PublicPages/SupCarlntro.html. Accessed October 7, 2016.

23. American Society of Clinical Oncology Annual Meeting. Cancer Care in Australia: Goals and Challenges for a Healthier Population. http://am.asco. org/cancer-care-australia-goals-and-challengeshealthier-population. Accessed October 7, 2016.

24. Xie XF. Research on the existing problems of clinical doctor-nurse collaboration. Chin J Modern Nurs. 2012;18:2509-2512. (in Chinese).

25. Qi YF. On the existing state of human resources in nursing and its countermeasures. World Latest Med Inf. 2014;14:543-543.

26. Wen GX, Mei HL. On KAP theory and voluntary unpaid blood donor recruitment. Chin J Blood Transfus. 2005;18:438-439. (in Chinese).

27. Lv RL. Effects of the nursing mode of knowledge and belief on the psychological status and quality of life of patients with breast cancer after operation. J Qilu Nurs. 2016;22:17-18. (in Chinese).

28. Tastan S, Hatipoglu S, Iyigun E, Kilic S. Implementation of a clinical pathway in breast cancer patients undergoing breast surgery. Eur $\mathrm{J}$ Oncol Nurs. 2012;16:368-374

29. Wu Y. Application of the safe nursing management model in radical breast cancer surgery patients. Chin J Clin Oncol Rehab. 2016;23:107-110. (in Chinese).

30. Fang Q. Effects of whole course professional case management in breast cancer nursing. Nurs $J$ Chin People's Liberation Army. 2013;2:51-54. (in Chinese).

31. Peng XL. Study on the effect of applying case management nursing model in nursing breast cancer patients. Today Nurse. 2015;10:57. (in Chinese).
32. Emery J. Cancer survivorship - the role of the GP. Australian Family Physician. 2014;43:521-525.

33. Allinson VM, Dent J. Supportive care after breast cancer surgery. Nurs Times. 2014;11:20-23.

34. Wang L. Application of the comfort care model in the nursing of perioperative period patients of breast cancer. Hosp Manag Forum. 2012;29:46-47. (in Chinese).

35. Liu PX, Hu AL, Li MR, Jin YS. Application of doubletrack continual health education model in postoperative rehabilitation of patients with breast cancer based on individualized and universalized perspectives. Nurs Pract Res. 2016;13:8-10. (in Chinese).

36. Eley R, Rogers C. Consumer perception of the effectiveness of a breast care nurse in providing holistic, coordinated care to women with breast cancer. Aust J Adv Nurs. 2012;29:56-61.

37. Storer L. Victorian Cancer Survivorship Program, Breast Cancer Survivorship; Integration with Community Practice. http://www.petermac.org/sites/default/ files/media-uploads/Breast_Cancer_Project.pdf. Accessed October 3, 2016.

38. Monash Health. Metastatic Breast Cancer Nurse Practitioner Model of Care Monash Health. http://www.health.vic.gov.au/_data/assets/ pdf_file/0011/918074/Monash-Health_MetastaticBreast-Cancer.pdf. Accessed October 6, 2016.

39. Meneses K, McNees P, Azuero A, Su XG, Hassey LA. Preliminary evaluation of psychoeducational support interventions on quality of life in rural breast cancer survivors post-primary treatment. Cancer Nurs. 2009;32:385-397.

40. Kuijpers W, Groen WG, Aaronson NK, van Harten WH. A systematic review of web-based interventions for patient empowerment and physical activity in chronic diseases: relevance for cancer survivors. J Med Internet Res. 2013;15:e37.

How to cite this article: Nie LT, Yan QY. Progress in research on the application of nursing models for breast cancer patients during the perioperative period. Front Nurs. 2018; 2: 83-90. https://doi.org/10.1515/fon-2018-0021 